\title{
$\beta$-Amino-n-butyric Acid Regulates Seedling Growth and Disease Resistance of Kimchi Cabbage
}

\author{
Yeong Chae Kim ${ }^{1 \dagger}$, Yeon Hwa Kim ${ }^{1 \dagger}$, Young Hee Lee ${ }^{1}$, Sang Woo Lee', Yun-Soek Chae', Hyun-Kyung Kang ${ }^{2}$, \\ Byung-Wook Yun ${ }^{3}$ and Jeum Kyu Hong ${ }^{1 *}$ \\ ${ }^{\prime}$ Department of Horticultural Science, Gyeongnam National University of Science and Technology (GNTech), Jinju 660-758, \\ Korea \\ ${ }^{2}$ Department of Environmental Landscape Architecture, Sangmyung University, Cheonan, Choongnam 330-720, Korea \\ ${ }^{3}$ Division of Plant Biosciences, School of Applied Biosciences, Kyungpook National University, Daegu 702-701, Korea \\ (Received on December 24, 2012; Revised on March 15, 2013; Accepted on March 29, 2013)
}

\begin{abstract}
Non-protein amino acid, $\beta$-amino- $n$-butyric acid (BABA), has been involved in diverse physiological processes including seedling growth, stress tolerance and disease resistance of many plant species. In the current study, treatment of kimchi cabbage seedlings with BABA significantly reduced primary root elongation and cotyledon development in a dose-dependent manner, which adverse effects were similar to the plant response to exogenous abscisic acid (ABA) application. BABA was synergistically contributing $A B A$-induced growth arrest during the early seedling development. Kimchi cabbage leaves were highly damaged and seedling growth was delayed by foliar spraying with high concentrations of BABA (10 to $20 \mathrm{mM}$ ). BABA played roles differentially in in vitro fungal conidial germination, mycelial growth and conidation of necrotroph Alternaria brassicicola causing black spot disease and hemibiotroph Colletotrichum higginsianum causing anthracnose. Pretreatment with BABA conferred induced resistance of the kimchi cabbage against challenges by the two different classes of fungal pathogens in a dose-dependent manner. These results suggest that $\mathrm{BABA}$ is involved in plant development, fungal development as well as induced fungal disease resistance of kimchi cabbage plant.
\end{abstract}

Keywords : $\beta$-amino- $n$-butyric acid, fungal development, induced disease resistance, kimchi cabbage, seedling development

Plants suffer from adverse ambient conditions such as pathogen attack and environmental stresses as they cannot move themselves away. To cope with these external stimuli, plants have developed sophisticated machinery in their

\footnotetext{
These authors contributed equally to this work.

*Corresponding author.

Phone) +82-55-751-3251, FAX) +82-55-751-3257

E-mail)jkhong@gntech.ac.kr
}

metabolism. One is 'preformed defense reaction', constitutively activated at a basal level prior to biotic and abiotic stresses (Osbourne, 1996). On the other hand, plant defense can be rapidly induced under stress conditions, and followed by activated calcium channel, complex defense signaling crosstalk (Garcia-Brugger et al., 2006), and accumulation of antimicrobial proteins (van Loon et al., 2006), that is called 'induced resistance (IR)'. Over the last few decades, practical efforts have been made to induce disease resistance of plants against pathogen infection in the laboratory and agricultural fields by exogenous applications of various defense activators, namely chemical elicitors. Salicylic acid (SA) and its derivative benzothiadiazole (BTH) are well known plant defense activators against invasions by obligate fungi and hemibiotrophic bacteria (Friedrich et al., 1996; Görlach et al., 1996; Vlot et al., 2009). BTH has been commercialized as an effective compound in the market by several manufacturers. Jasmonic acid (JA), fatty acid-derived compound confers plant tolerance to necrotrophic fungi and herbivores (Abe et al., 2009; Rowe et al., 2010). JA occasionally acts antagonistically with SA-dependent defense signalling during plant-pathogen interactions (Smith et al., 2009). However, application of JA followed by SA significantly enhanced disease resistance to Cucumber mosaic virus (Luo et al., 2011). In recent years, plant growth-promoting rhizobacteria (PGPR) and mycorrhizal fungi have been considered as promising biotic agents for modern plant protection, because these microbes could enhance disease resistance of plants to various challenging pathogens (van Wees et al., 2008).

$\beta$-Amino- $n$-butyric acid (BABA), a non-protein amino acid, is known as defense priming molecule that potentiates accelerated defense to pathogen infection and osmotic stress. BABA-induced resistance (BABA-IR) has been frequently found in many crops against diverse bacterial and fungal species in recent decades (Hamiduzzaman et al., 2005; Olivieri et al., 2009; Walz and Simon, 2009). With an array 
of example of BABA-IR, the mode-of-action of BABA including crosstalk with other defense signaling pathways in plants remains largely unknown. BABA-IR in Arabidopsis against Pectobacterium carotovorum ssp. carotovorum was independent of SA, JA and ethylene-mediated defense signaling (Chen et al., 2012). SA is required for BABA-IR of potato against late blight disease by Phytophthora infestans (Eschen-Lippold et al., 2010). Molecular genetics analysis using model plant Arabidopsis revealed that BABA-IR to necrotrophic fungi Alternaria brassicicola and Plectospharella cucumerina infection was dependent on abscisic acid (ABA)-mediated signaling pathways (Ton and Mauch-Mani, 2004). By contrast, ABA-independent pathway for BABA-IR also was suggested in lettuce against Bremia lactucae, downy mildew oomycete pathogen (Cohen et al., 2010). It is widely well known that ABA is a closely related plant hormone controlling abiotic stresses like high salinity, drought and low temperature (Nakashima et al., 2009; Tuteja, 2007). Indeed, treatment with BABA can trigger both abiotic and biotic stress tolerance in Arabidopsis. Faster and stronger defense gene expressions were observed in BABA-primed plant cells in response to microbial infection and environmental stresses (Hamiduzzaman et al., 2005; Jakab et al., 2005; Zimmerli et al., 2000). Accelerated defense gene expression by BABA priming was likely to render immediate sensitization to adverse plant environment conditions. These observations suggest that $\mathrm{BABA}$ interacts with different signaling pathways for triggering plant defenses.

A few recent intriguing emerging findings have suggested novel actions of BABA in plant development and growth (Jakab et al., 2001; Wu et al., 2010; Zimmerli et al., 2008). While exogenous BABA application influenced silique length and seed production (Jakab et al., 2001), it did not affect seed germination of Arabidopsis compared to the water-treated control seeds (Wu et al., 2010). In addition, primary root elongation and rosette leaf growth were severely retarded in the BABA-treated Arabidopsis plants, accompanying increased accumulation of anthocyanin and gene expression of chalcone synthase (CHS) and dihydroflavonol-4-reductase (DFR) responsible for anthocyanin biosynthetic pathway. Interestingly, BABA-mediated Arabidopsis root growth inhibition was significantly increased by additive $\mathrm{ABA}$ application, but partially rescued by L-glutamine treatment (Wu et al., 2010).

Kimchi cabbage (Brassica rapa subsp. pekinensis), cruciferous plant like Arabidopsis, is one of the most important economic vegetable crops in Asia, evidenced by genome sequence recently publicly launched (The Brassica rapa Genome Sequencing Consortium, 2011). Although BABA can be one of the important regulators for plant growth and stress tolerance, no report on the role of BABA is demon- strated in kimchi cabbage. In this study, biological responses of phytopathogenic fungi and kimchi cabbage seedlings to BABA treatment were evaluated in the light of development and disease resistance. Furthermore, our data suggest that a physiological interaction of BABA with phytohormone $\mathrm{ABA}$ is also operated, at least in part, in kimchi cabbage.

\section{Materials and Methods}

Plant growth. Kimchi cabbage (B. rapa var. glabra cv. Samrack-eolgari) seeds were sterilized with $1 \%$ sodium hypochlorite $(\mathrm{NaOCl})$ for $10 \mathrm{~min}$ and then washed 5 times with sterile distilled water. Seeds were germinated on the water-saturated paper for 1 day. Six germinated seeds were sown in one plastic square pot $(12.5 \mathrm{~cm} \times 8 \mathrm{~cm} \times 6 \mathrm{~cm})$ containing steam-sterilized soil mixtures. The kimchi cabbage plants were raised in a growth room under the controlled environments of $23 \pm 2^{\circ} \mathrm{C}$ and $70 \mu$ mole photons/ $\mathrm{m}^{2} / \mathrm{s}$ illumination with $12 \mathrm{~h}$ light $/ 12 \mathrm{~h}$ dark photoperiod at the $60 \%$ relative humidity until used. To measure fresh weight $(\mathrm{FW})$ and primary root elongation on the synthetic media for early seedling development, surface-sterilized germinating seeds with radicle (1 $\mathrm{mm}$ in length) were placed onto Murashige-Skoog (MS) agar media (Murashige and Skoog, 1962) containing different BABA and ABA doses. The MS agar plates were placed vertically during the kimchi cabbage seedling growth.

Chemical treatments. To investigate effect of BABA on the growth of kimchi cabbage seedlings, different concentrations of BABA $(0,0.1,0.2,0.5,1,2,5$ and $10 \mathrm{mM})$ prepared in distilled water were foliar-sprayed onto 2week-old kimchi cabbage seedlings, and then FW of the leaves was measured. Two-week-old seedlings were also gently removed from the potting mixtures, and transferred to the solutions with increasing concentrations of BABA in $2 \mathrm{~mL}$-microtubes. FW of the seedlings were measured before and after transferring to the BABA solutions.

For BABA-IR of kimchi cabbage plants to fungal infections, the same concentration range of the BABA solution was foliar-sprayed 1 day prior to fungal inoculations of $A$. brassicicola and $C$. higginsianum.

Different concentrations of BABA $(0,0.001,0.01,0.1,1$ and $10 \mathrm{mM})$ and $\mathrm{ABA}(0,0.5,1,2,5$ and $10 \mu \mathrm{M})$ were added into half strength of MS media for the early seedling development. BABA solutions $(0,5,10,15$ and $20 \mathrm{mM})$ were foliar-sprayed onto 2-week-old kimchi cabbage seedlings, and FW of the seedlings were measured 7 days after BABA treatment to observe BABA effect on kimchi cabbage seedling growth.

In vitro fungal pathogen growth in response to BABA 
treatment. Conidia of $A$. brassicicola (Mycothèque de l'Université catholique de Louvain, MUCL 20297) and $C$. higginsianum isolate C97-28 (Korean Agricultural Culture Collection, KACC 40807) were harvested from the 6-dayold culture on V8 juice agar and potato dextrose agar (PDA) media, respectively. Conidial suspension $\left(2 \times 10^{5}\right.$ conidia $/ \mathrm{mL}$ ) was mixed with different concentrations of BABA. Drops were incubated on slide glass in the moist chamber without cover glass at $25^{\circ} \mathrm{C}$ for $5 \mathrm{~h}$. Conidial germination was observed under light microscope and relative germination rate by BABA treatment was calculated compared to that in BABA-untreated control. Conidial germination was evaluated when conidia without BABA treatment germinated ca. $80 \%$.

$A$. brassicicola and $C$. higginsianum were cultured on PDA media supplemented with different concentrations of $\operatorname{BABA}(0,0.1,0.2,0.5,1,2,5$ and $10 \mathrm{mM})$. The PDA plates with mycelial plugs placed at the center were incubated at $25^{\circ} \mathrm{C}$ under darkness. Diameters of the growing fungal colonies were measured and relative mycelial growths were expressed as percentage compared to that on the untreated media.

For investigation on conidation of the two fungi onto the BABA-containing PDA media, mycelial discs $(5 \mathrm{~mm}$ in diameter) were removed from the fungal colonies 7 days after cultures and transferred to microtubes. Sterile distilled water $(0.1 \mathrm{~mL})$ was added to the tubes and mixed vigorously to prepare conidial suspension. Conidia in the suspension were counted under the light microscope. Conidation was expressed as conidia no. $/ \mathrm{cm}^{2}$ of media area.

Fungal inoculations and disease evaluations. $A$. brassicicola was grown on $\mathrm{V} 8$ juice agar media at $25^{\circ} \mathrm{C}$ in the dark. Conidia were obtained from 7 day-old cultures by gentle scarping and conidial suspension in sterile water was prepared by filtering through two layers of sterile cheesecloth. Concentration of conidial suspension was adjusted to $5 \times$ $10^{4}$ conidia/mL supplemented with $0.05 \%$ Tween 20 as a surfactant. Primary and secondary leaves of kimchi cabbage plants at 2-week-old stage were inoculated by spraying conidial suspension, or by placing four $7 \mu \mathrm{L}$ droplets of conidial suspensions of $A$. brassicicola. The inoculated plants were transferred to moist chamber for $48 \mathrm{~h}$ under dark condition to allow symptom to develop. To quantify BABA-IR, number of spreading susceptible necrotic lesions on the drop-inoculated leaves was counted. Disease severity of kimchi cabbage seedlings by the fungal inoculation was visibly observed for conidial suspension-sprayed plants.

C. higginsianum was also cultured on PDA at $25^{\circ} \mathrm{C}$ under darkness. Preparation of conidial suspension of $C$. higginsianum was the same with those performed for $A$. brassicicola. Conidial suspension of $1 \times 10^{4}$ conidia $/ \mathrm{mL}$ supplemented with $0.05 \%$ Tween 20 were adjusted for $C$. higginsianum to inoculate the kimchi cabbage leaves. The inoculated plants were placed in moist chamber for $24 \mathrm{~h}$ with darkness for symptom development. Evaluation of BABA-IR against $C$. higginsianum was performed with same procedures used for BABA-IR against $A$. brassicicola.

To observe the fungal symptom development on the kimchi cabbage plants, 4 pots containing 6 seedlings/pot were used for BABA-treated seedlings and untreated ones.

Statistical analyses. Analysis of variance (ANOVA) was used to determine the effects of treatments on disease resistance and growth of kimchi cabbage plants. Means were compared using Duncan's multiple range tests. Statistical analysis was performed with the SAS version 8.1 (SAS Institute, Inc., Cary. NC).

\section{Results}

Effect of BABA on seedling growth of kimchi cabbage. Early seedling development of kimchi cabbage was arrested by increasing concentration of BABA treatment (Fig. 1). Cotyledon expansion, root emergence and root hair formation were severely retarded during germination 1 day after high dose of BABA treatment (Fig. 1A), shown as reduced FW and primary root length 3 days after treatment (Fig. 1B). Total FW and root elongation of the seedlings treated with more than $1 \mathrm{mM}$ of BABA were significantly decreased. Treatment of germinating seedlings with $1 \mathrm{mM}$ and $10 \mathrm{mM}$ of BABA reduced their FW by $19 \%$ and $63 \%$, respectively. Primary root elongation of the BABA-treated germinating seedlings was also retarded to 82 and $21 \%$ by 1 and $10 \mathrm{mM}$ of BABA treatments, respectively.

Plant hormone, ABA, is involved in root growth. High dose of exogenously applied ABA led to reduced growth of primary root in many plant species (Lin and Kao, 2001; Newton, 1997; Zhang et al., 2007). We investigated whether root growth of kimchi cabbage seedlings were also affected by ABA and relationship with BABA-mediated root growth retardation. Treatment of germinating kimchi cabbage seeds with ABA delayed growth of the seedlings, showing reduced FW and elongation of primary root (Fig. 1C). FW of kimchi cabbage seedlings were drastically reduced by increasing concentration of exogenous $\mathrm{ABA}$ at least by $0.5 \mathrm{mM}$ to $84.4 \%$, compared to that of untreated seedlings. Root growth was decreased to $25.4 \%$ compared to the control seedlings at a concentration of $10 \mathrm{mM}$ ABA. In addition to $1 \mathrm{mM}$ of ABA, BABA application synergistically affected seedlings growth with loss of FW and root elongation (Fig. 1D).

BABA-mediated plant growth arrest and tissue damages. 
A
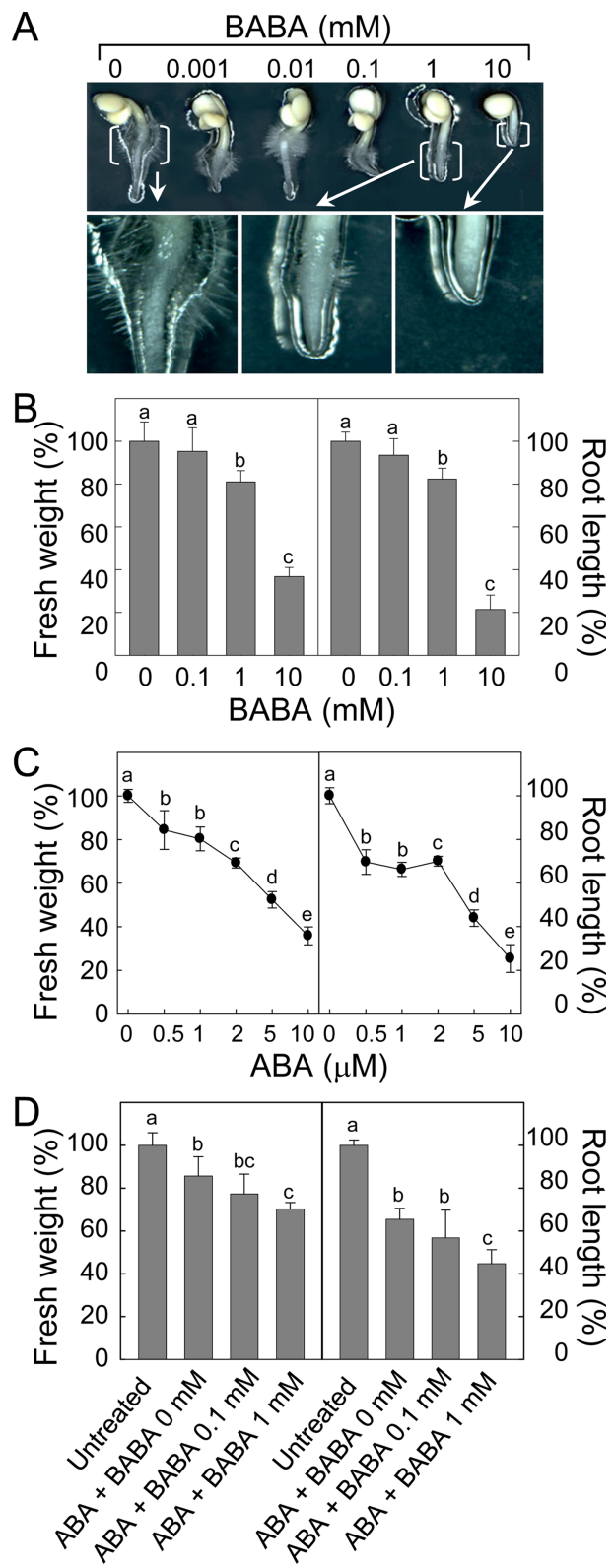

Fig. 1. Early seedling development of kimchi cabbage in response to $\beta$-amino- $n$-butyric acid (BABA) treatment. (A) Dose-dependent growth retardation of kimchi cabbage seedling. Photo was taken 1 day after BABA treatment of germinated seeds. (B) Fresh weight and root elongation of kimchi cabbage seedlings 3 days after different concentrations of BABA treatment. (C) Kimchi cabbage seedling growth in response to increasing concentration of abscisic acid (ABA). Fresh weight and primary root elongation of the seedlings were measured 3 days after ABA treatment. (D) The seedling growth in response to BABA treatment in combination with ABA. Fresh weight and primary root elongation of the seedlings were measured 2 days after the chemical treatment. Fresh weight and primary root length of the seedlings were measured. Data are the means \pm standard errors from four independent experiments performed with similar results $(n=8)$. Mean separation by Duncan's multiple range test at $P=0.05$. The same letter above bars represented no significant difference between treatments.
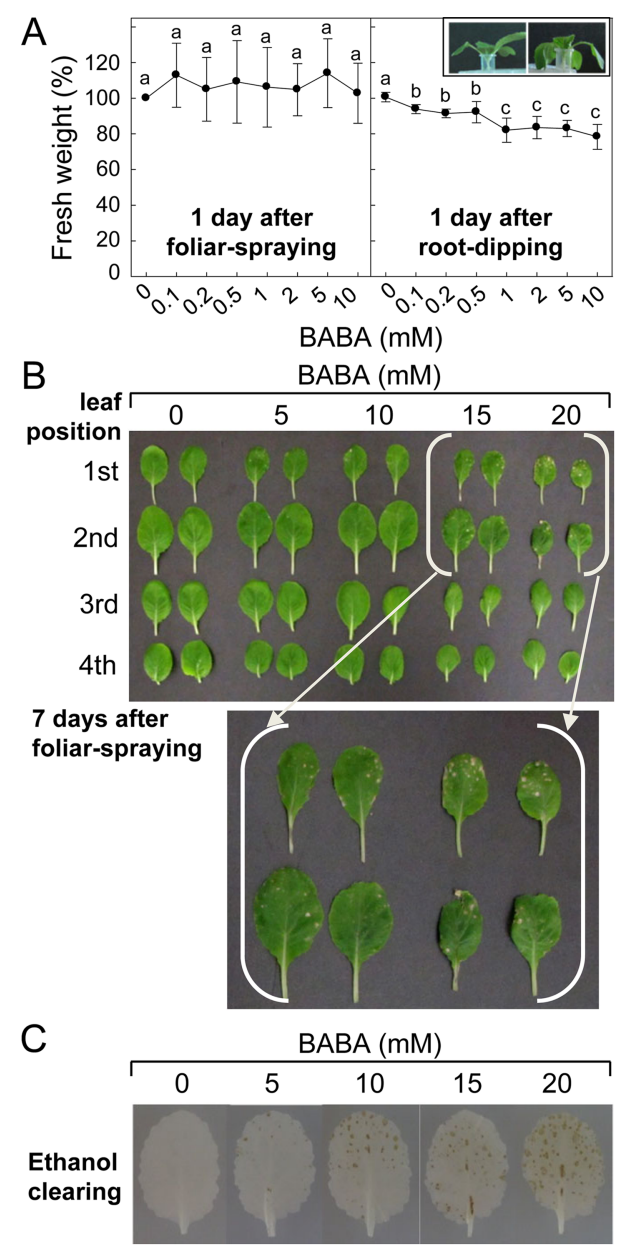

$\mathrm{D}$

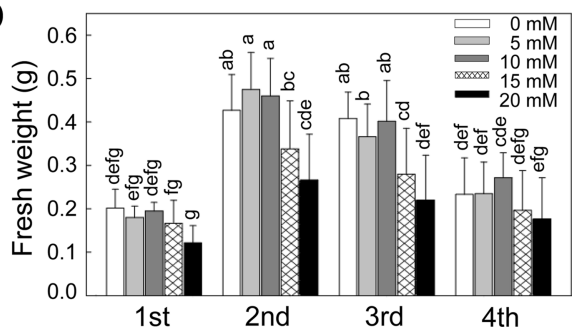

Fig. 2. Effect of $\beta$-amino- $n$-butyric acid (BABA) treatment on seedlings growth and leaf tissue damage of kimchi cabbage. (A) The seedlings growth by foliar-spraying and root-dipping with BABA. Fresh weight of the seedlings 1 day after treatment was measured. Normal (left) and wilting (right) of the seedlings treated with 0 and $10 \mathrm{mM}$ of BABA, respectively, by root-dipping were demonstrated in inset. Photo was taken 1 day after treatment. (B) Leaf damages of the kimchi cabbage seedlings by foliar-spraying of high dose of BABA. Photo was taken 7 days after treatment. Leaf positions were countered from the bottom of the kimchi cabbage seedling. (C) BABA-treated kimchi cabbage leaves that chlorophylls were removed with ethanol overnight to clearly visualize tissue damage. Necrotic lesions were seen as leaf spots of brown color. (D) Fresh weight of leaves at different positions at 7 days after BABA treatment. Data are the means \pm standard deviations from four independent experiments. Mean separation by Duncan's multiple range test at $P=0.05$. The same letter above bars represented no significant difference between treatments. 
Application of BABA on 2-week-old kimchi cabbage seedlings affected plant growth and tissue damages (Fig. 2). The kimchi cabbage seedlings were foliar-sprayed or root-dipped into different concentration of BABA, and the plant growth was investigated 1 day after BABA treatment by measuring FW (Fig. 2A). We could not find any significant changes in FW of the seedlings treated with foliar-sprayed BABA (0.1 to $10 \mathrm{mM}$ ), while increasing BABA doses by root-dipping led to adverse effect on the seedling growth with wilting and reduced FW.

Relatively higher dose of BABA (5, 10, 15 and $20 \mathrm{mM})$ was applied by foliar spraying and plant growth was investigated 7 days after the treatment (Fig. 2B-D). Kimchi cabbage seedlings at 2-week-old have fully expanded primary and secondary leaves, and third leaf began to emerge. When foliar part of the seedlings was treated with BABA, 15 and $20 \mathrm{mM}$ of BABA caused tissue damages 7 days after treatment, but relatively lower concentrations of BABA did not lead to any visible tissue damage. No foliar damages were found in the newly developing 3rd and 4th leaves 7 days after BABA treatment. BABA-triggered tissue damage of the leaves was clearly shown by removing chlorophylls with ethanol (Fig. 2C). Tissue damages in the leaves treated with relatively lower dose of BABA (5 - 10 $\mathrm{mM}$ ), not evidently visible in the leaves without the clearing, were also seen by ethanol clearing. Enlarged brown necrotic areas in the leaves treated with higher BABA (15$20 \mathrm{mM}$ ) were distinctly demonstrated by the clearing. FW of the kimchi cabbage seedlings was measured to quantify plant growth affected by BABA treatment (Fig. 2D). FW of the 1st leaves was not affected by different concentrations of BABA, and FW of 2nd leaves was reduced only by 20 $\mathrm{mM}$ of BABA. The 15 and $20 \mathrm{mM}$ of BABA decreased FW of the 3rd leaves. There was no difference in the FW of 4th leaves by all concentrations of BABA tested.

Effect of BABA on in vitro fungal growth. In vitro conidial germination of $A$. brassicicola and $C$. higginsianum was modulated by exogenous BABA treatment (Fig. 3A). Lower BABA dose (0.1-0.2 mM) slightly arrested conidial germination of $A$. brassicicola, but the germination rate was not altered by $0.5 \mathrm{mM}$ of BABA. Interestingly, $1 \mathrm{mM}$ of BABA rather enhanced conidial germination transiently, however, the germination frequency at more than $2 \mathrm{mM}$ of BABA was returned to the control level. By contrast, increasing dose of BABA accelerated conidial germination of $C$. higginsianum. The conidial germination was being improved in a dose-dependent manner from $1 \mathrm{mM}$ up to 10 $\mathrm{mM}$ BABA. By the concentration of $10 \mathrm{mM}$, the conidial germination was significantly increased by $120.3 \%$ compared to that of untreated conidia.

Mycelial growth of $A$. brassicicola and C. higginsianum
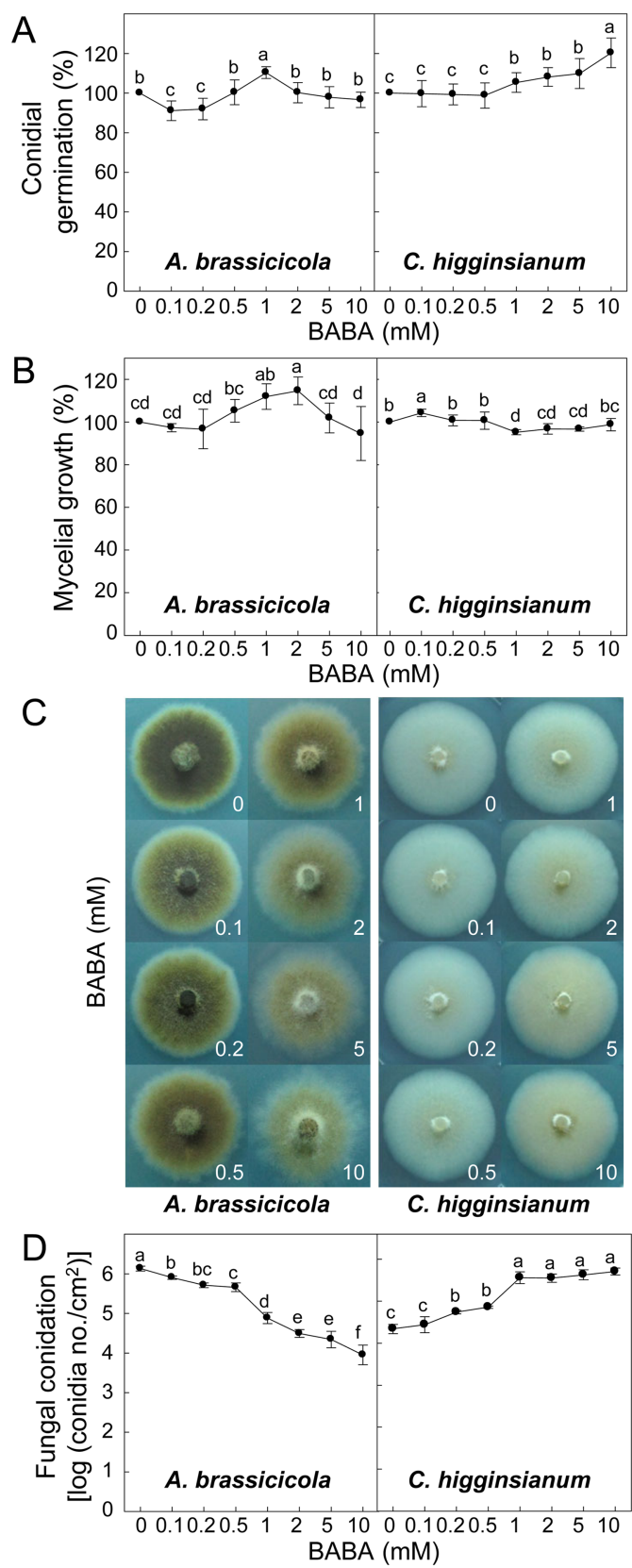

Fig. 3. Effect of BABA on in vitro growth of phytopathogenic fungi, A. brassicicola and C. higginsianum. (A) In vitro conidial germination and (B) mycelial growth of the fungi treated with increasing concentrations of BABA. Data are the means \pm standard deviations from four independent experiments. Mean separation by Duncan's multiple range test at $P=0.05$. The same letter above bars represented no significant difference between treatments. (C) Fungal colonies formed on PDA media supplemented with different concentrations of BABA. Photos were taken 7 days after culture under dark conditions at $25^{\circ} \mathrm{C}$. (D) Conidation of $A$. brassicicola and $C$. higginsianum modulated by BABA treatment. Data are the mean conidial number \pm standard deviations from four independent experiments. Mean separation by Duncan's multiple range test at $P=0.05$. The same letter above bars represented no significant difference between treatments. 

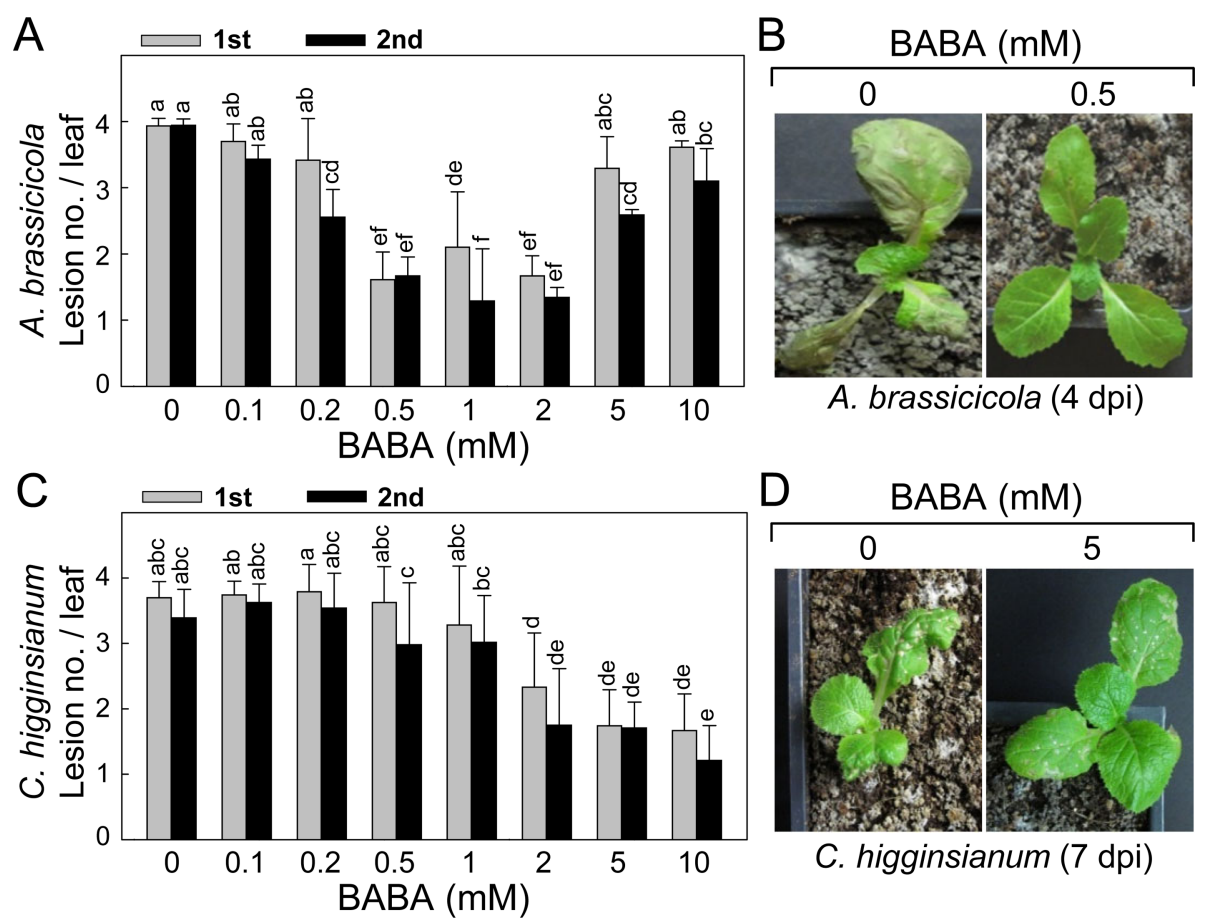

Fig. 4. BABA-induced disease resistance of kimchi cabbage plants to black spot by necrotrophic fungal $A$. brassicicola and anthracnose by hemibiotrophic fungal $C$. higginsianum. (A) Reduced black spot symptom development on the leaves of kimchi cabbage seedlings by BABA pretreatment. Different concentrations of BABA were evenly sprayed onto the seedlings 1 day prior to fungal inoculation by placing 4 drops of conidial suspension on the first (1st) and second (2nd). Induced resistance was expressed as lesion no./leaf. (B) BABAinduced disease resistance of kimchi cabbage seedlings against $A$. brassicicola infection. The seedlings were pretreated with or without BABA 1 day prior to foliar spray inoculation. Symptom development was observed and photos were taken at 4 days post-inoculation (dpi). (C) Reduced anthracnose symptom development on the leaves of kimchi cabbage seedlings by BABA pretreatment. Different concentrations of BABA were evenly sprayed onto the seedlings 1 day prior to fungal inoculation by placing 4 drops of conidial suspension on the first (1st) and second (2nd). Induced resistance was expressed as lesion no./leaf. (D) BABA-induced disease resistance of kimchi cabbage seedlings against $C$. higginsianum infection. The seedlings were pretreated with or without BABA 1 day prior to foliar spray inoculation. Symptom development was observed, and photos were taken at 7 dpi. Number of necrotic lesions on the first and second leaves was counted. Data are the means \pm standard deviations from four independent experiments. Mean separation by Duncan's multiple range test at $P=0.05$. The same letter above bars represented no significant difference between treatments.

on BABA-containing media was evaluated by measuring colony diameter of the two fungal species to investigate whether BABA has in vitro antifungal activities against vegetative growth of the two fungi (Fig. 3B). Diameter of fungal colonies formed on the PDA media supplemented with different concentration of BABA was measured at 7 days after fungal cultures of $A$. brassicicola and $C$. higginsianum at $25^{\circ} \mathrm{C}$. Treatment with 0.1 to $0.5 \mathrm{mM}$ of BABA did not affect mycelial growth of $A$. brassicicola, but $1-2$ $\mathrm{mM}$ of BABA slightly increased the fungal growth. Much higher dose of BABA $(5-10 \mathrm{mM})$ rather did not influence mycelial growth of $A$. brassicicola compared to mycelial growth in the untreated control. Surprisingly, dark olive colored A. brassicicola colony in the untreated media became faint by increasing BABA concentration (Fig. 3C). Mycelial growth of $C$. higginsianum was slightly increased by $0.1 \mathrm{mM}$ of BABA, but $0.2-0.5 \mathrm{mM}$ did not increase the growth any more. One to $5 \mathrm{mM}$ of BABA rather decreased the mycelial growth, but $10 \mathrm{mM}$ of BABA did not alter the growth. Increasing BABA changed colony made salmoncolored and reduced aerial mycelium formation.

Conidation of the $A$. brassicicola and $C$. higginsianum on PDA media was also influenced by BABA treatment (Fig. 3D). Increasing BABA concentration drastically decreased conidation of A. brassicicola, whereas $C$. higginsianum was stimulated in conidation.

BABA-induced fungal disease resistance in kimchi cabbage plants. Pretreatment of kimchi cabbage plants with BABA 1 day prior to fungal inoculation differentially conferred resistance against two different fungal diseases, black spot disease and anthracnose caused by $A$. brassicicola and C. higginsianum, respectively (Fig. 4). To evaluate BABAIR quantitatively, 1st and 2nd leaves were drop-inoculated with conidial suspensions of the two fungi.

BABA-IR appeared in the A. brassicicola-inoculated 
leaves of kimchi cabbage plants in a BABA dose-dependent manner (Fig. 4A). In the 1st leaves, lesion number per leaf was not changed by $0-0.2 \mathrm{mM}$ of BABA, whereas 0.5 $-2 \mathrm{mM}$ of BABA reduced lesion number effectively. Much higher concentrations of BABA $(5-10 \mathrm{mM})$ did not confer any enhanced resistance to the fungal infection. In the 2nd leaves, $0.2 \mathrm{mM}$ of BABA began to decrease the lesion number, and $0.5-2 \mathrm{mM}$ was most effective concentration range of BABA. BABA-IR by $5 \mathrm{mM}$ was slightly lower than those triggered by $0.5-2 \mathrm{mM}$ of BABA concentrations. By $10 \mathrm{mM}$ of BABA, the efficiency of disease protection mediated was diminished, showing relatively lower induced resistance than that accomplished by $0.2-2$ $\mathrm{mM}$ of BABA pretreatment.

After fungal inoculation by foliar spraying conidial suspension of $A$. brassicicola, the 1st true leaves of the inoculated plants were severely damaged and blighted at 4 days post-inoculation (dpi) without BABA pretreatment (Fig. 4B). Necrotic and circular spot lesions appeared on the 2 nd and 3rd leaves of the seedlings. Lesions on the leaves were enlarged and coalesced over time. By contrast, susceptible spreading leaf spots by the fungal infection were hardly detected in the challenge-inoculated leaves pretreated with $0.5 \mathrm{mM}$ of BABA.

BABA-IR against $C$. higginsianum inoculation was also shown in the kimchi cabbage plants (Fig. 4C). Increasing concentration of BABA was correlated with reduction in lesion formation by anthracnose. BABA pretreatment in the range of $0.1-1 \mathrm{mM}$ could not reduce anthracnose both in the $1 \mathrm{st}$ and 2 nd leaves. More than $2 \mathrm{mM}$ of BABA efficiently decreased anthracnose symptom development in the leaves, in which no difference in the level of IR was found.

Foliar inoculation of kimchi cabbage seedlings using conidial suspension of $C$. higginsianum caused leaf blight of primary leaves. While numerous necrotic lesions appeared in the inoculated 2nd and 3rd leaves (Fig. 4D), pretreatment with $5 \mathrm{mM}$ of BABA reduced number and size of lesions showing tiny scattered lesions the inoculated leaves of kimchi cabbage seedlings at 7 dpi.

\section{Discussion}

Non-protein amino acid BABA became first known as a priming agent for induced disease resistance in many crops belonged to diverse genus including cucumber, grape, pepper and tomato plants (Cohen et al., 1994; Hamiduzzaman et al., 2005; Hong et al., 1999; Jeun and Park, 2003). Knowledge of mode-of-action of BABA in plant tissues during primed disease resistance is still largely limited. Although BABA can be a promising agent for enhanced disease resistance demonstrated in many plant species, underlying mechanism(s) of BABA particularly connecting between plant development and defense perspectives has not been studied.

BABA as a developmental regulator of kimchi cabbage plants. BABA has been shown to involve in developmental regulation of several plants. High dose of BABA affected adversely seed germination of pearl millet, whereas seed germination of Arabidopsis was not inhibited (Shailasree et al., 2001; Zimmerli et al., 2008). BABA treatment of kimchi cabbage seeds also could not affect germination in this study (data not shown). We examined effect of BABA during early seedling development of kimchi cabbage plants. Significantly reduced early seedling development of kimchi cabbage occurred by exogenous BABA application during primary root growth and cotyledon expansion. ABA is a phytohormone controlling seed dormancy, stomatal closure, root elongation and adaptation to environmental stresses in plants (Zeevaart and Creelman, 1998). In the present study, ABA was also negatively involved in early seedling development of kimchi cabbage plant, especially primary root elongation. Treatment with ABA suppressed root elongation of kimchi cabbage seedlings. ABA may interact with exogenous $\mathrm{BABA}$ in the kimchi cabbage seedlings, showing that BABA and ABA synergistically inhibit seedling development. Interaction of BABA with ABA signaling was demonstrated in plant development and disease resistance of Arabidopsis. BABA could compromise A. brassicicola-reduced ABA accumulation and increased ABSCISIC ACID-INSENSITIVE1 (ABI1) gene expression in Arabidopsis (Flors et al., 2008). However, ABA was not involved in BABA-induced disease resistance found in lettuce plants against downy mildew oomycete $B$. lactucae evidenced by that $\mathrm{ABA}$ did not affect the disease resistance alone and in combination with BABA (Cohen et al., 2010).

BABA-triggered tissue damages of kimchi cabbage plants. Two-week-old kimchi cabbage seedlings treated with BABA showed different response dependent on application method. BABA-mediated retardation of kimchi cabbage seedling developments can be due to phytotoxic effects. While foliar-spraying of BABA did not cause visible phytotoxic effect on kimchi cabbage seedlings 1 day after treatment, the treatment by root-dipping with same range of concentrations turned the seedlings severely wilted. It may be due to low permeability of BABA through leaf surfaces but efficient translocation from roots to proximal leaf part as demonstrated in lettuce and tomato plants (Cohen and Gisi, 1994; Cohen et al., 2010). By foliarspraying, relatively higher BABA amount was required for induced resistance of lettuce plants to downy mildew than BABA-IR established by soil-drenching (Cohen et al., 2010). Responses of 2-week-old seedlings treated with high con- 
centration of BABA by foliar-spraying were investigated 7 days after treatment. The 1st and 4th leaves were not affected in FW, but FW of 2nd and 3rd leaves was significantly reduced. It indicates that BABA-induced growth retardation occurs only in the developing and emerging leaves, but not in already fully expanded developed leaves (1st) and in the leaves not directly treated (4th). High dose $(10-20 \mathrm{mM})$ of BABA-induced tissue damages were found in the 1st and 2nd leaves of kimchi cabbage seedlings. It was found that superoxide anion $\left(\mathrm{O}_{2}^{-}\right)$and hydrogen peroxide $\left(\mathrm{H}_{2} \mathrm{O}_{2}\right)$ accumulation as histochemically observed in tobacco leaves treated with BABA (Siegrist et al., 2000). However, generation of superoxide anion $\left(\mathrm{O}_{2}^{-}\right)$and hydrogen peroxide $\left(\mathrm{H}_{2} \mathrm{O}_{2}\right)$ was not detected in the BABA-treated kimchi cabbage leaves by histochemical staining (data not shown). ROS production is not likely to relate with the BABAmediated cell death in the kimchi cabbage. Further studies on cellular and biochemical relations of BABA-triggered tissue damages remain to be investigated in kimchi cabbage plants.

Role of BABA during in vitro fungal growth. Since BABA-IR was first found in tomato plant to late blight, in vitro antimicrobial activity of BABA has been rarely observed (Cohen et al., 1994). Recently, increasing evidences on the direct antimicrobial activity of BABA against plant pathogens have been demonstrated, although BABA has still ability to confer enhanced resistance via defense priming machinery. Even 2 and $8 \mathrm{mM}$ of BABA have arrested mycelial growth and conidial germination of Leptosphaeria maculans, respectively, and more than $12 \mathrm{mM}$ of BABA was demonstrated to induce disease protection in Brassica napus against blackleg by L. maculans (Šašek et al., 2012). In other cases, relatively higher concentrations of BABA inhibited spore germination and germ tube elongation of Penicillium digitatum and P. italicum in vitro (Porat et al., 2003; Tavallali et al., 2008). Mycelial growth and conidial germination of Botrytis cinerea were also restrained by increasing BABA doses (Fischer et al., 2009). However, BABA-IR in many plants including apple fruits was still thought be achieved as a non-antimicrobial component (Reuveni et al., 2003).

In this study, we aimed to investigate whether BABA has direct antifungal activities against kimchi cabbage pathogens. In vitro conidial germination and mycelial growth of two different fungal species $A$. brassicicola and $C$. higginsianum treated with BABA were evaluated. Surprisingly, in vitro conidial germination of the two fungi was differentially regulated. Different range of BABA concentrations positively or negatively affected the conidial germination of $A$. brassicicola and $C$. higginsianum, respectively. Relatively lower BABA (1 mM) dose induced conidial germination of
A. brassicicola, whereas rapid conidial germination of $C$. higginsianum occurred by increasing dose of BABA up to $10 \mathrm{mM}$. Fungal spore germination have been stimulated or inhibited by endogenous and/or exogenous stimuli from the ambient environment (Macko et al., 1977; Tsurushima et al., 1995). Amino acid alanine promoted spore germination of Rhizopus oligosporus but proline negatively affected the spore germination (Thanh et al., 2005). Amino acids such as serine, alanine, glutamic acid, arginine and methionine inhibited conidial germination of A. alternata, whereas aspartic acid, phenylalanine and threonine stimulated the germination (Migahed and Nofel, 2001). The present study on in vitro regulation of the conidial germination suggests that metabolic process of non-protein amino acid BABA within the fungal cells during the germination also remains investigated.

In vitro mycelial growth of $A$. brassicicola was accelerated by 1 to $2 \mathrm{mM}$ of BABA. Alternaria spp. are taxonomically classified as family Dematiaceae with their conidia and hyphae melanized and pigmented in normal growth conditions (Pridham and Woodhead, 1977; Thomma, 2003). BABA treatment may adversely affect melanization of $A$. brassicicola in vitro shown by changing colony color faint. Melanin production of $A$. alternata is critically important in conidial development as well as fungal pathogenicity (Kawamura et al., 1997, 1999). Although molecular genetics studies on pigmentation of $A$. brassicicola have been not demonstrated so far, $\mathrm{BABA}$ treatment is likely to reduce melanin production of $A$. brassicicola and the virulence on kimchi cabbage seedlings. However, A. brassicicola still seems to have pathogenicity to kimchi cabbage treated with high dose of BABA.

Mycelial growth of $C$. higginsianum in vitro was not drastically regulated by exogenous BABA treatment. However, colony of $C$. higginsianum on the culture media supplemented with increasing BABA turned to be sticky salmon-colored, implying production of conidial mass on the media. C. gloeosporioides produce salmon-colored conidial mass on the artificial media and the infected host tissues (Choi and Hong, 2009; Choi et al., 2011). Conidia from lesions of the infected host tissues can be disseminated to uninoculated plant parts or other plants as a secondary inoculum for the disease progression in the field. Conidation of $C$. higginsianum was increased in vitro by BABA treatment in this study. In vitro and in planta fungal sporulation were known to be influenced by external stimuli, i.e. light, aeration, $\mathrm{pH}$, injury to the culture, nutritional conditions, as well as host factors (Dahlberg and van Etten, 1982; Rotem et al., 1978). Information on BABA-induced in vitro conidation of $C$. higginsianum is still limited.

$\mathrm{BABA}$ is an isomer of $\alpha$-aminobutyric acid (AABA) and $\gamma$-aminobutyric acid (GABA). AABA was known as one of 
the good nitrogen sources for conidial production of Venturia inaequalis, apple scab fungus (Ross, 1968). GABA plays a role in nitrogen metabolism to regulate carbon-to-nitrogen $(\mathrm{C}: \mathrm{N})$ balance and in protections against oxidative and osmotic stresses in plants (Bouché and Fromm, 2004). GABA can be used as a major nitrogen source for phytopathogenic fungus during pathogenesis. High increase in GABA content was found in tomato leaves inoculated by virulent Cladosporium fulvum, tomato leaf mold fungus (Solomon and Oliver, 2001). GABA was suggested to be utilized by the C. fulvum in the tomato leaf apoplast as a nutrient source (Solomon and Oliver, 2002). Carbon-tonitrogen ratio of $15: 1$ in the nutritional media enhanced conidation of Colletotrichum spp. compared to relative carbon-to-nitrogen ratios 40:1 or 5:1 (Jackson and Bothast, 1990). BABA treatment may influence on nutritional balance and promote conidation of $C$. higginsianum. On the other hand, high concentration of non-protein amino acid BABA can merely trigger amino acid stresses controlling of the fungi, as demonstrated in Arabidopsis plant treated with BABA showing anthocyanin accumulation caused by general amino acid stress (Wu et al., 2010). Involvement of BABA in fungal nutritional balance, amino acid metabolism, in vitro conidation and pathogenicity of $A$. brassicicola and C. higginsianum remains elucidated.

To investigate whether BABA induce resistance to the pathogen attack, we applied different concentrations of BABA onto the kimchi cabbage seedlings 1 day prior to challenge inoculation by $A$. brassicicola and $C$. higginsianum. It is still not known how much BABA is entered into plant tissues from the leaf surface after spraying and whether it directly contacts with fungal conidia and/or mycelia on and/or within leaf tissues 1 day after treatment.

BABA-primed disease resistance of kimchi cabbage in planta. IR in the kimchi cabbage plants against the two fungal pathogens was shown. A range of $0.5-2 \mathrm{mM}$ BABA was sufficient to mediate IR to A. brassicicola in the kimchi cabbage. Jakab et al. (2001) has briefly mentioned BABAIR of two Brassica species, broccoli (B. oleracea var. italica) and cauliflower (B. oleracea var. botrytis) to A. brassicicola infections, but BABA-IR of the kimchi cabbage plant to $A$. brassicicola has not been reported. BABA treatment compromised the enhanced disease susceptibility in the camalexin-deficient Arabidopsis mutant pad3-1 highly susceptible to A. brassicicola infection (Ton and Mauch-Mani, 2004). BABA-IR was demonstrated in Brassica juncea against Alternaria brassicae, another fungal species causing Alternaria leaf black spot (Kamble and Bhargava, 2007). Treatment with BABA efficiently controlled moldy-core decay of apple fruits by $A$. alternata without direct inhibition of conidial germination and mycelial growth (Reuveni et al.,
2003). These results suggest that BABA can activate enhanced disease resistance of many host plants to different Alternaria spp. Thus this report will be first to demonstrate dose-dependent chemical BABA-induced disease resistance to $A$. brassicicola in kimchi cabbage plants.

Interestingly, relatively higher dose of BABA (10 mM) could confer minute IR in the kimchi cabbage plants to $A$. brassicicola. These might be due to BABA causing phytotoxicity in the 1st and 2nd leaves of kimchi cabbage plants by more than $5 \mathrm{mM}$ concentration (Fig. 2A), at which FW of the leaves was significantly not affected, but tissue damages of small specks appeared on the leaves. Similar dose-dependent BABA-IR effect was found in grapefruit inoculated by green mold $P$. digitatum (Porat et al., 2003). Twenty $\mathrm{mM}$ of BABA effectively triggered disease resistance, however, less effective at either higher or lower concentrations of BABA. These indicated that optimal concentration of $\mathrm{BABA}$ is required for inducing disease resistance in plants including kimchi cabbage plants to combat pathogen attacks efficiently.

BABA-IR was differently effective in the 1st and 2nd leaves of kimchi cabbage plants against $A$. brassicicola infection. It was demonstrated that BABA-IR was more efficiently expressed in the 2nd leaves than in the 1 st leaves. BABA concentration of $0.5-2 \mathrm{mM}$ triggered IR in the $1 \mathrm{st}$ leaves, while broader range of $0.2-10 \mathrm{mM}$ BABA was effective in the 2nd leaves. BABA-IR in tobacco plants to downy mildew oomycete pathogen Peronospora tabacina was dependent on the leaf position demonstrating that effect of BABA was relatively low in the lower leaves than upper leaves (Cohen, 1994). Disease protection against late blight $P$. infestans by BABA occurred in leaf positiondependently as well as dose-dependently in tomato plants (Cohen and Gisi, 1994). These findings support the notion that expression of BABA-IR was developmentally modulated in the plants.

BABA-mediated disease protection occurs in many plant species against a wide range of pathogens including fungi and bacteria, which promoted us to evaluate broad disease resistance by BABA in kimchi cabbage plants as well. Unlike necrotrophic attack by $A$. brassicicola during pathogenesis, hemibiotrophic fungus $C$. higginsianum invades host plant based on initial biotrophic and subsequent necrotrophic stages, which have been also frequently observed in penetrating Colletotrichum spp. like C. graminicola on maize and $C$. truncatum on pea (Münch et al., 2008; Narusaka et al., 2004; O'Connell et al., 1993). BABA-IR against Colletotrichum spp. was previously described in cucumber and pepper plants to C. orbiculare and C. coccodes, respectively (Hong et al., 1999; Jeun and Park, 2003). Despite transition of $C$. higginsianum from biotrophy to necrotrophy at the later infection stage, high dose of BABA- 
mediated phytotoxicity did not affect BABA-IR. It seems that the BABA-triggered tissue was healed and no more nutrition supply for fungal growth. Interestingly, effective dosage for BABA-IR against $C$. higginsianum was different from that against $A$. brassicicola in kimchi cabbage. BABAIR against $A$. brassicicola was achieved by some range of concentrations, whereas BABA-IR against $C$. higginsianum was more effective by higher concentrations. It may be due to difference in infection style of the two fungal species. Necrotrophic fungi, such as $A$. brassicicola and $B$. cinerea utilized nutrition derived from necrotizing and dead plant tissues for their ingress and colonization in the host tissues (Govrin and Levine, 2000; Mayer et al., 2001). High dose of BABA resulted in phytotoxicity to kimchi cabbage seedlings showing formation of tiny specks on the leaves, which may cause plant derived nutritional release for accelerated fungal development and compromised BABA-IR in the kimchi cabbage plants. Development-regulated BABAIR against $A$. brassicicola infection was not found in the $C$. higginsianum-inoculated kimchi cabbage leaves.

For mode-of-action of BABA as a defense priming agent against pathogen attacks, enhanced activation of defense gene expression, callose deposition, phytoalexin production and cell wall lignifications have been investigated in several plant species (Hamiduzzaman et al., 2005; Olivieri et al., 2009; Ton and Mauchi-Mani, 2004). Defense priming and signaling cross-talk related to $\mathrm{BABA}$ during IR against $A$. brassicicola and $C$. higginsianum infections in kimchi cabbage plants needs to be explained further through molecular and genetic approaches. In addition, BABA-IR in kimchi cabbage plants against broad spectrum of plant pathogens including virus, oomycetes, bacteria and nematodes remains investigated as well.

Taken together, BABA was found to be developmental regulator as well as priming agent for induced disease resistance against fungal infections in kimchi cabbage plants. Molecular and genetic studies on the signal cross-talk of various plant growth regulators and defense components with BABA during seedling development and induced disease resistance in kimchi cabbage will provide more valuable information on mode-of-action of BABA in plants.

\section{Acknowledgments}

We are grateful to Dr. Sang Hee Kim (Indiana University, USA) for helpful discussion and critical review of our manuscript. This research was supported by grants from The Agricultural Genome Center (TAGC), in the NextGeneration BioGreen21 Program (grant no. PJ008211 to Jeum Kyu Hong), Rural Development Administration, Republic of Korea, and also partially supported by Gyeongnam National University of Science and Technology Grant
2013 to Jeum Kyu Hong.

\section{References}

Abe, H., Shimoda, T., Ohnishi, J., Kugimiya, S., Narusaka, M., Seo, S., Narusaka, Y., Tsuda, S. and Kobayashi, M. 2009. Jasmonate-dependent plant defense restricts thrips performance and preference. BMC Plant Biol. 9:97.

Bouché, N. and Fromm, H. 2004. GABA in plants: just a metabolite? Trends Plant Sci. 9:110-115.

Chen, P.-W., Singh, P. and Zimmerli, L. 2012. Priming of the Arabidopsis pattern-triggered immunity response upon infection by necrotrophic Pectobacterium carotovorum bacteria. Mol. Plant Pathol. 14:58-70.

Choi, K. O. and Hong, J. K. 2009. First report of anthracnose occurrence on sloumi by Colletotrichum gloeosporioides in Korea. Plant Pathol. J. 25:434.

Choi, O., Seo J., Kwon, J.-H. and Kim, J. 2011. Anthracnose caused by Colletotrichum gloeosporioides on sweet crabapple in Korea. Plant Pathol. J. 27:396.

Cohen, Y. 1994. 3-Aminobutyric acid induces systemic resistance against Peronospora tabacina. Physiol. Mol. Plant Pathol. 44:273-288.

Cohen, Y. and Gisi, U. 1994. Systemic translocation of ${ }^{14} \mathrm{C}-\mathrm{DL}-3-$ aminobutyric acid in tomato plants in relation to induced resistance against Phytophthora infestans. Physiol. Mol. Plant Pathol. 45:441-456.

Cohen, Y., Niderman, T., Mösinger, E. and Fluhr, R. 1994. $\beta$ Aminobutyric acid induces the accumulation of pathogenesisrelated proteins in tomato (Lycopersicon esculentum L.) plants and resistance to late blight infection caused by Phytophthora infestans. Plant Physiol. 104:59-66.

Cohen, Y., Rubin, A. E. and Kilfin, G. 2010. Mechanisms of induced resistance in lettuce against Bremia lactucae by DL$\beta$-amino-butyric acid (BABA). Eur. J. Plant Pathol. 126:553573.

Dahlberg, K. R. and van Etten, J. L. 1982. Physiology and biochemistry of fungal sporulation. Annu. Rev. Phytopathol. 20:281-301.

Eschen-Lippold, L., Altmann, S. and Rosahl, S. 2010. DL- $\beta-A m i-$ nobutyric acid-induced resistance of potato against Phytophthora infestans requires salicylic acid but not oxylipins. Mol. Plant-Microbe Interact. 23:585-592.

Fischer, M. J. C., Farine, S., Chong, J., Guerlain, P. and Bertsch, C. 2009. The direct toxicity of BABA against grapevine ecosystem organisms. Crop Protect. 28:710-712.

Flors, V., Ton, J., van Doorn, R., Jakab, G., García-Agustín, P. and Mauch-Mani, B. 2008. Interplay between JA, SA and ABA signaling during basal and induced resistance against Pseudomonas syringae and Alternaria brassicicola. Plant J. 54:81-92.

Friesrich, L., Lawton, K., Ruess, W., Masner, P., Specker, N., Rella, M. G., Meier, B., Dincher, S., Staub, T., Uknes, S., Métraux, J. P., Kessmann, H. and Ryals, J. 1996. A benzothiadiazole derivatives induces systemic acquired resistance in tobacco. Plant J. 10:61-70. 
Garcia-Brugger, A., Lamotte, O., Vandelle, E., Bourque, S., Lecourieux, D., Poinssot, B., Wendehenne, D. and Pugin, A. 2006. Early signaling events induced by elicitors of plant defenses. Mol. Plant-Microbe Interact. 7:711-724.

Görlach, J., Volrath, S., Knauf-Beiter, G., Hengu, G., Beckhove, U., Kogel, K. H., Staub, M. T., Ward, E., Kessmann, H. and Ryals, J. 1996. Benzothiadiazole, a novel class of inducers of systemic acquired resistance, activates expression and disease resistance in wheat. Plant Cell 8:629-643.

Govrin, E. M. and Levine, A. 2000. The hypersensitive response facilitates plant infection by the necrotrophic pathogen Botrytis cinerea. Curr. Biol. 10:751-757.

Hamiduzzaman, M. M., Jakab, G., Barnavon, L., Neuhaus, J. M. and Mauch-Mani, B. 2005. $\beta$-Amino-butyric acid-induced resistance against downy mildew in grapevine acts through the potentiate of callose formation and jasmonic acid signaling. Mol. Plant-Microbe Interact. 18:819-829.

Hong, J. K., Hwang, B. K. and Kim, C. H. 1999. Induction of local and systemic resistance to Colletotrichum coccodes in pepper plants by DL- $\beta$-amino- $n$-butyric acid. J. Phytopathol. 147:193-198.

Jackson, M. A. and Bothast, R. J. 1990. Carbon concentration and carbon-to-nitrogen ratio influence submerged-culture conidation by the potential bioherbicide Colletotrichum truncatum NRRL 13737. Appl. Environ. Microbiol. 56:3435-3438.

Jakab, G., Cottier, V., Toquin, V., Rigoli, G., Zimmerli, L., Métraux, J. P. and Mauch-Mani, B. 2001. $\beta$-Aminobutyric acid-induced resistance in plants. Eur. J. Plant Pathol. 107:29-37.

Jakab, G., Ton, J., Flors, V., Zimmerli, L., Métraux, J. P. and MauchMani, B. 2005. Enhancing Arabidopsis salt and drought stress tolerance by chemical priming for its abscisic acid responses. Plant Physiol. 139:267-274.

Jeun, Y. C. and Park, E. W. 2003. Ultrastructures of the leaves of cucumber plants treated with DL-3-aminobutyric acid at the vascular bundle and the penetration sites after inoculation with Colletotrichum orbicularae. Plant Pathol. J. 19:85-91.

Kamble, A. and Bhargava, S. 2007. $\beta$-Aminobutyric acid-induced resistance in Brassica juncea against the necrotrophic pathogen Alternaria brassicae. J. Phytopathol. 155:152-158.

Kawamura, C., Moriwaki, J., Kimura, N., Fujita, Y., Fuji, S., Hirano, T., Koizumi, S. and Tsuge, T. 1997. The melanin biosynthesis genes of Alternaria alternata can restore pathogenicity of the melanin-deficient mutants of Magnaporthe grisea. Mol. Plant-Microbe Interact. 10:446-453.

Kawamura, C., Tsujimoto, T. and Tsuge, T. 1999. Targeted disruption of a melanin biosynthesis gene affects conidial development and UV tolerance in the Japanese pear pathotype of Alternaria alternata. Mol. Plant-Microbe Interact. 12:59-63.

Lin, C. C. and Kao, C. H. 2001. Abscisic acid changes in cell wall peroxidase activity and hydrogen peroxide level in roots of rice seedlings. Plant Sci. 160:323-329.

Luo, Y., Shang, J., Zhao, P., Xi, D., Yuan, S. and Lin, H. 2011. Application of jasmonic acid followed by salicylic acid inhibits Cucumber mosaic virus replication. Plant Pathol. J. 27:5358.

Macko, V., Trione, E. J. and Young, S. A. 1977. Identification of the germination self-inhibitor from uredospores of Puccinia striiformis. Phytopathology 67:1473-1474.

Mayer, A. M., Staples, R. C. and Gil-ad, N. L. 2001. Mechanisms of survival of necrotrophic fungal plant pathogens in hosts expressing the hypersensitive response. Phytochemistry 58: 33-41.

Migahed, F. F. and Nofel, A. M. 2001. Leaf exudates of Vicia faba and their effects on Botrytis fabae and some associated fungi. Mycobiology 24:198-204.

Münch, S., Lingner, U., Floss, D. S., Ludwig, N., Sauer, N. and Deising, H. B. 2008. The hemibiotrophic lifestyle of Colletotrichum species. J. Plant Physiol. 165:41-51.

Murashige, T. and Skoog, F. 1962. A revised medium for rapid growth and bioassays with tobacco cultures. Physiol. Plant. 15:474-497.

Nakashima, K., Ito, Y. and Yamaguchi-Shinozaki, K. 2009. Transcriptional regulatory networks in response to abiotic stresses in Arabidopsis and grasses. Plant Physiol. 149:88-95.

Narusaka, Y., Narusaka, M., Park, P., Kubo, Y., Hirayama, T., Seki, M., Shiraishi, T., Ishida, J., Nakashima, M., Enju, A., Sakurai, T., Satou, M., Kobayashi, M. and Shinozaki, K. 2004. $R C H 1$, a locus in Arabidopsis that confers resistance to the hemibiotrophic fungal pathogen Colletotrichum higginsianum. Mol. Plant-Microbe Interact. 17:749-762.

Newton, E. J. 1977. Abscisic acid effects on fronds and roots of Lemna minor L. Amer. J. Bot. 64:45-49.

O’Connell, R. J., Uronu, A. B., Waksman, G., Nash, C., Keon, J. P. R. and Bailey, J. A. 1993. Hemibiotrophic infection of Pisum sativum by Colletotrichum truncatum. Plant Pathol. 42:774-783.

Olivieri, F. P., Lobato, M. C., Altamiranda, E. G., Daleo, G. R., Huarte, M., Guevara, M. G. and Andreu, A. B. 2009. BABA effects on the behavior of potato cultivars infected by Phytophthora infestans and Fusaium solani. Eur. J. Plant Pathol. 123:47-56.

Osbourne, A. E. 1996. Preformed antimicrobial compounds and plant defense against fungal attack. Plant Cell 8:1821-1831.

Porat, R., Vinokur, V., Weiss, B., Cohen, L., Daus, A., Goldschmidt, E. E. and Droby, S. 2003. Induction of resistance to Penicillium digitatum in grapefruit by $\beta$-aminobutyric acid. Eur. J. Plant Pathol. 109:901-907.

Pridham, J. B. and Woodhead, S. 1977. The biosynthesis of melanin in Alternaria. Phytochemistry 16:903-906.

Reuveni, M., Sheglove, D. and Cohen, Y. 2003. Control of moldycore decay in apple fruits by $\beta$-aminobutyric acids and potassium phosphites. Plant Dis. 87:933-936.

Ross, R. G. 1968. Amino acids as nitrogen sources for conidial production of Venturia inaequalis. Can. J. Bot. 46:1555-1560.

Rotem, J., Cohen, Y. and Bashi, E. 1978. Host and environmental influences on sporulation in vivo. Annu. Rev. Phytopathol. 16:83-101.

Rowe, H. C., Walley, J. W., Corwin, J., Chan, E. K. F., Dehesh, K. and Kliebenstein, D. J. 2010. Deficiencies in jasmonate-mediated plant defense reveal quantitative variation in Botrytis cinerea pathogenesis. PLoS Pathog. 6:e1000861.

Šašek, V., Nováková, M., Dobrev, P. I., Valentová and Burketová, 
L. 2012. $\beta$-aminobutyric acid protects Brassica napus plants from infection by Leptosphaeria maculans. Resistance induction or a direct antifungal effect? Eur. J. Plant Pathol. 133:279-289.

Shailasree, S., Sarosh, B. R., Vasanthi, N. S. and Shetty, H. S. 2001. Seed treatment with $\beta$-aminobutyric acid protects Pennisetum glaucum systemically from Sclerospora graminicola. Pest Manag. Sci. 57:721-728.

Siegrist, J., Orober, M. and Buchenauer, H. 2000. $\beta$-Aminobutyric acid-mediated enhancement of resistance in tobacco to tobacco mosaic virus depends on the accumulation of salicylic acid. Physiol. Mol. Plant Pathol. 56:95-106.

Smith, J. L., de Moraes, C. M. and Mescher, M. C. 2009. Jasmonate- and salicylate-mediated plant defense responses to insect herbivores, pathogens and parasitic plants. Pest Manag. Sci. 65:497-503.

Solomon, P. S. and Oliver, R. P. 2001. The nitrogen content of the tomato leaf apoplast increases during infection by Cladosporium fulvum. Planta 213:241-249.

Solomon, P. S. and Oliver, R. P. 2002. Evidence that $\gamma$-aminobutyric acid is a major nitrogen source during Cladosporium fulvum infection of tomato. Planta 214:414-420.

Tavallali, V., Karimi, S., Mohammadi, S. and Hojati, S. 2008. Effects of $\beta$-aminobutyric acid on the induction of resistance to Penicillium italicum. World Appl. Sci. J. 5:345-351.

Thanh, N. V., Rombouts, F. M. and Nout, M. J. R. 2005. Effect of individual amino acids and glucose on activation and germination of Rhizopus oligosporus sporangiospores in tempe starter. J. Appl. Microbiol. 99:1204-1214.

The Brassica rapa Genome Sequencing Consortium 2011. The genome of the mesopolyploid crop species Brassica rapa. Nature Genet. 43:1035-1039.

Thomma, B. P. H. J. 2003. Alternaria spp.: from general saprophyte to specific parasite. Mol. Plant Pathol. 4:225-236.

Ton, J. and Mauch-Mani, B. 2004. $\beta$-Amino-butyric acid-induced resistance against necrotrophic pathogen is based on ABA- dependent priming for callose. Plant J. 38:119-130.

Tsurushima, T., Ueno, T., Fukami, H., Irie, H. and Inoue, M. 1995. Germination self-inhibitors from Colletotrichum gloeosporioides f. sp. jussiaea. Mol. Plant-Microbe Interact. 8:652-657.

Tuteja, N. 2007. Abscisic acid and abiotic stress signaling. Plant Sig. Behav. 2:135-138.

van Loon, L. C., Rep, M. and Pierterse, C. M. J. 2006. Significance of inducible defense related proteins in infected plants. Annu. Rev. Phytopathol. 44:135-162.

van Wees, S. C. M., Van der Ent, S. and Pieterse, C. M. J. 2008. Plant immunity responses triggered by beneficial microbes. Curr. Opin. Plant Biol. 11:443-448.

Vlot, A. C., Dempsey, D. M. A. and Klessig, D. F. 2009. Salicylic acid, a multifaceted hormone to combat disease. Annu. Rev. Phytopathol. 47:177-206.

Walz, A. and Simon, O. 2009. $\beta$-Amino-butyric acid-induced resistance in cucumber against biotrophic and necrotrophic pathogens. J. Phytopathol. 157:356-361.

Wu, C. C., Singh, P., Chen, M. C. and Zimmerli, L. 2010. LGlutamine inhibits beta-aminobutyric acid-induced stress resistance and priming in Arabidopsis. J. Exp. Bot. 61: 9951002.

Zeevaart, J. A. D. and Creelman, R. A. 1998. Metabolism and physiology of abscisic acid. Annu. Rev. Plant Physiol. Plant Mol. Biol. 39:439-473.

Zhang, Y., Yang, C., Li, Y., Zheng, N., Chen, H., Zhao, Q., Gao, T., Guo, H. and Xie, Q. 2007. SDIR1 is a RING finger E3 ligase that positively regulates stress-responsive abscisic acid signaling in Arabidopsis. Plant Cell 19:1912-1929.

Zimmerli, L., Hou, B. H., Tsai, C. H., Jakab, G., Mauch-Mani, B. and Somerville, S. 2008. The xenobiotic $\beta$-aminobutyric acid enhances Arabidopsis thermotolerance. Plant J. 53:144-156.

Zimmerli, L., Jakab, G., Métraux, J. P. and Mauch-Mani, B. 2000. Potentiation of pathogen-specific defense mechanisms in Arabidopsis by $\beta$-aminobutyric acid. Proc. Natl. Acad. Sci. USA 97:12920-12925. 